In April 27th 2000 issue of Nature

\title{
A Flat Universe from High-Resolution Maps of the Cosmic Microwave Background Radiation
}

\author{
P. de Bernardis ${ }^{1}$, P.A.R.Ade ${ }^{2}$, J.J.Bock ${ }^{3}$, J.R.Bond ${ }^{4}$, J.Borrill ${ }^{5,6}$, A.Boscaleri ${ }^{7}$, K.Coble ${ }^{8}$, \\ B.P.Crill ${ }^{9}$, G.De Gasperis $^{1}$ 0, P.C.Farese ${ }^{8}$, P.G.Ferreira ${ }^{11}$, K.Ganga ${ }^{9,1} 2$, M.Giacometti ${ }^{1}$, E.Hivon ${ }^{9}$, \\ V.V.Hristov ${ }^{9}$, A.Iacoangeli ${ }^{1}$, A.H.Jaffe ${ }^{6}$, A.E.Lange ${ }^{9}$, L.Martinis ${ }^{13}$, S.Masi ${ }^{1}$, P.Mason $^{9}$, \\ P.D.Mauskopf ${ }^{14,15}$, A.Melchiorri ${ }^{1}$, L.Miglio ${ }^{16}$, T.Montroy ${ }^{8}$, C.B.Netterfield ${ }^{16}$, E.Pascale $^{7}$, \\ F.Piacentini ${ }^{1}$, D.Pogosyan ${ }^{4}$, S.Prunet ${ }^{4}$, S.Rao $^{17}$, G.Romeo $^{17}$, J.E.Ruhl ${ }^{8}$, F.Scaramuzzi ${ }^{13}$, \\ D.Sforna ${ }^{1}$, N.Vittorio ${ }^{10}$ \\ 1) Dipartimento di Fisica, Universitá di Roma La Sapienza, P.le A. Moro 2, 00185 Roma, Italy, \\ 2) Department of Physics, Queen Mary and Westfield College, Mile End Road, London, E1 4NS,
} UK,

3) Jet Propulsion Laboratory, Pasadena, CA, USA,

4) CITA University of Toronto, Canada,

5) NERSC-LBNL, Berkeley, CA, USA,

6) Center for Particle Astrophysics, University of California at Berkeley, 301 Le Conte Hall, Berkeley CA 94720, USA,

7) IROE - CNR, Via Panciatichi 64, 50127 Firenze, Italy,

8) Department of Physics, University of California at Santa Barbara, Santa Barbara, CA 93106, USA,

9) California Institute of Technology, Mail Code: 59-33, Pasadena, CA 91125, USA,

10) Dipartimento di Fisica, Universitá di Roma Tor Vergata, Via della Ricerca Scientifica 1, 00133 Roma, Italy,

11) Astrophysics, University of Oxford, Keble Road, OX1 3RH, UK,

12) PCC, College de France, 11 pl. Marcelin Berthelot, 75231 Paris Cedex 05, France,

13) ENEA Centro Ricerche di Frascati, Via E. Fermi 45, 00044 Frascati, Italy, 
14) Physics and Astronomy Dept, Cardiff University, UK,

15) Dept of Physics and Astronomy, U.Mass. Amherst, MA, USA,

16) Departments of Physics and Astronomy, University of Toronto, Canada,

17) Istituto Nazionale di Geofisica, Via di Vigna Murata 605, 00143, Roma, Italy,.

The blackbody radiation left over from the Big Bang has been transformed by the expansion of the Universe into the nearly isotropic 2.73 K Cosmic Microwave Background. Tiny inhomogeneities in the early Universe left their imprint on the microwave background in the form of small anisotropies in its temperature. These anisotropies contain information about basic cosmological parameters, particularly the total energy density and curvature of the universe. Here we report the first images of resolved structure in the microwave background anisotropies over a significant part of the sky. Maps at four frequencies clearly distinguish the microwave background from foreground emission. We compute the angular power spectrum of the microwave background, and find a peak at Legendre multipole $\ell_{\text {peak }}=(197 \pm 6)$, with an amplitude $D T_{200}=(69 \pm 8) \mu K$. This is consistent with that expected for cold dark matter models in a flat (euclidean) Universe, as favoured by standard inflationary scenarios.

Photons in the early Universe were tightly coupled to ionized matter through Thomson scattering. This coupling ceased about 300,000 years after the Big Bang, when the Universe cooled sufficiently to form neutral hydrogen. Since then, the primordial photons have travelled freely through the universe, redshifting to microwave frequencies as the universe expanded. We observe those photons today as the cosmic microwave background (CMB). An image of the early Universe remains imprinted in the temperature anisotropy of the CMB. Anisotropies on angular scales larger than $\sim 2^{\circ}$ are dominated by the gravitational redshift the photons undergo as they leave the density fluctuations present at decoupling ${ }^{(1,2)}$. Anisotropies on smaller angular scales are enhanced by oscillations of the photon-baryon fluid before decoupling ${ }^{(3)}$. These oscillations are driven by the primordial density fluctuations, and their nature depends on the matter content of 
the universe.

In a spherical harmonic expansion of the CMB temperature field, the angular power spectrum specifies the contributions to the fluctuations on the sky coming from different multipoles, each corresponding to the angular scale $\theta=\pi / \ell$. Density fluctuations over spatial scales comparable to the acoustic horizon at decoupling produce a peak in the angular power spectrum of the CMB, occurring at multipole $\ell_{\text {peak }}$. The exact value of $\ell_{\text {peak }}$ depends on both the linear size of the acoustic horizon and on the angular diameter distance from the observer to decoupling. Both these quantities are sensitive to a number of cosmological parameters (see for example ref.4), but $\ell_{\text {peak }}$ primarily depends on the total density of the Universe, $\Omega_{0}$. In models with a density $\Omega_{0}$

near $1, \ell_{\text {peak }} \sim 200 / \Omega^{1 / 2}$. A precise measurement of peak can efficiently constrain the density and thus the curvature of the Universe. Observations of CMB anisotropies require extremely sensitive and stable instruments. The $\mathrm{DMR}^{5}$ instrument on the COBE satellite mapped the sky with an angular resolution of $\sim 7^{\circ}$, yielding measurements of the angular power spectrum at multipoles $\ell<20$. Since then, experiments with finer angular resolution ${ }^{6-16}$ have detected CMB fluctuations on smaller scales and have produced evidence for the presence of a peak in the angular power spectrum at peak $\ell_{\text {peak }} \sim 200$.

Here we present high resolution, high signal-to-noise maps of the CMB over a significant fraction of the sky, and derive the angular power spectrum of the CMB from $\ell=50$ to 600 . This power spectrum is dominated by a peak at multipole $\ell_{\text {peak }}=(197 \pm 6)(1 \sigma$ error $)$. The existence of this peak strongly supports inflationary models for the early universe, and is consistent with a flat, Euclidean Universe.

\section{The Instrumrent}

The BOOMERanG (Balloon Observations Of Millimetric Extragalactic Radiation and Geomagnetics) experiment is a microwave telescope that is carried to an altitude of $\sim 38 \mathrm{~km}$ by a balloon. BOOMERanG combines the high sensitivity and broad frequency coverage pioneered by an earlier generation of balloon-borne experiments with the long ( 10 days $)$ integration time 
available in a long-duration balloon flight over Antarctica. The data described here were obtained with a focal plane array of 16 bolometric detectors cooled to $0.3 \mathrm{~K}$. Single-mode feedhorns provide two $18^{\prime}$ full-width at half-maximum (FWHM) beams at $90 \mathrm{GHz}$ and two $10^{\prime}$ (FWHM) beams at $150 \mathrm{GHz}$. Four multi-band photometers each provide a 10.5, 14 and 13 FWHM beam at 150, 240 and $400 \mathrm{GHz}$ respectively. The average in-flight sensitivity to CMB anisotropies was 140, 170, 210 and $2700 \mu K \cdot s^{1 / 2}$ at $90,150,240$ and $400 \mathrm{GHz}$ respectively. The entire optical system is heavily baffled against terrestrial radiation. Large sun-shields improve rejection of radiation from $>60^{\circ}$ in azimuth from the telescope boresight. The rejection has been measured to be greater than $80 \mathrm{~dB}$ at all angles occupied by the Sun during the CMB observations. Further details on the instrument can be found in refs $17-21$.

\section{Observation}

BOOMERanG was launched from McMurdo Station (Antarctica) on 29 December 1998, at 3:30 GMT. Observations began 3 hours later, and continued uninterrupted during the 259-hour flight. The payload approximately followed the $79^{\circ} \mathrm{S}$ parallel at an altitude that varied daily between 37 and $38.5 \mathrm{~km}$, returning within $50 \mathrm{~km}$ of the launch site.

We concentrated our observations on a target region, centred at roughly right ascension (RA) $5 \mathrm{~h}$, declination (dec.) $-45^{\circ}$, that is uniquely free of contamination by thermal emission from interstellar dust ${ }^{(22)}$ and that is approximately opposite the Sun during the austral summer. We mapped this region by repeatedly scanning the telescope through $60^{\circ}$ at fixed elevation and at constant speed. Two scan speeds $\left(1^{\circ} \cdot \mathrm{s}^{-1}\right.$ and $2^{\circ} \cdot \mathrm{s}^{-1}$ in azimuth) were used to facilitate tests for systematic effects. As the telescope scanned, degree-scale variations in the CMB generated sub-audio frequency signals in the output of the detector ${ }^{(23)}$. The stability of the detetector system was sufficient to allow sensitive measurements on angular scales up to tens of degrees on the sky. The scan speed was sufficiently rapid with respect to sky rotation that identical structures were observed by detectors in the same row in each scan. Detectors in different rows observed the same structures delayed in time by a few minutes. 
At intervals of several hours, the telescope elevation was interchanged between $40^{\circ}, 45^{\circ}$ and $50^{\circ}$ in order to increase the sky coverage and to provide further systematic tests. Sky rotation caused the scan centre to move and the scan direction to rotate on the celestial sphere. A map from a single day at a single elevation covered roughly $22^{\circ}$ in declination and contained scans rotated by $\pm 11^{\circ}$ on the sky, providing a cross-linked scan pattern. Over most of the region mapped, each sky pixel was observed many times on different days, both at $1^{\circ} \cdot \mathrm{s}^{-1}$ and $2^{\circ} \cdot \mathrm{s}^{-1}$ scan speed, with different topography, solar elongation and atmospheric conditions, allowing strong tests for any contaminating signal not fixed on the celestial sphere.

The pointing of the telescope has been reconstructed with an accuracy of $2^{\prime}$ r.m.s.using data from a Sun sensor and rate gyros. This precision has been confirmed by analyzing the observed positions of bright compact HII regions in the Galactic plane (RCW38 ${ }^{24}$, RCW57, IRAS08576 and IRAS1022) and of radio-bright point sources visible in the target region (the QSO 0483-436, the BL-Lac object 0521-365 and the blazar 0537-441).

\section{Calibrations}

The beam pattern for each detector was mapped before flight using a thermal source. The main lobe at 90, 150 and $400 \mathrm{GHz}$ is accurately modelled by a Gaussian function. The $240 \mathrm{GHz}$ beams are well modelled by a combination of two Gaussians. The beams have small shoulders (less than $1 \%$ of the total solid angle), due to aberrations in the optical system. The beam-widths were confirmed in flight via observations of compact sources. By fitting radial profiles to these sources we determine the effective angular resolution, which includes the physical beamwidth and the effects of the $2^{\prime}$ r.m.s.pointing jitter. The effective FWHM angular resoluti on of the $150 \mathrm{GHz}$ data that we use here to calculate the CMB power spectrum is $(10 \pm 1)^{\prime}$, where the error is dominated by uncertainty in the pointing jitter.

We calibrated the 90, 150 and $240 \mathrm{GHz}$ channels from their measured response to the CMB dipole.

The dipole anisotropy has been accurately $(0.7 \%)$ measured by COBE-DMR ${ }^{25}$, fills the beam and has the same spectrum as the CMB anisotropies at smaller angular scales, making it the ideal 
calibrator for CMB experiments. The dipole signal is typically $\sim 3 \mathrm{mK}$ peak-to-peak in each $60^{\circ}$ scan, much larger than the detector noise, and appears in the output of the detectors at $\mathrm{f}=0.008 \mathrm{~Hz}$ and $\mathrm{f}=0.016 \mathrm{~Hz}$ in the $1^{\circ} \cdot \mathrm{s}^{-1}$ and $2^{\circ} \cdot \mathrm{s}^{-1}$ scan speeds, respectively. The accuracy of the calibration is dominated by two systematic effects: uncertainties in the low-frequency transfer function of the electronics, and low-frequency, scan-synchronous signals. Each of these is significantly different at the two scan speeds. We found that the dipole-fitted amplitudes derived from separate analysis of the $1^{\circ} \cdot \mathrm{s}^{-1}$ and $2^{\circ} \cdot \mathrm{s}^{-1}$ data agree to within $\pm 10 \%$ for every channel, and thus we assign a $10 \%$ uncertainty in the absolute calibration.

\section{From detector signals to CMB maps}

The time-ordered data comprises $5.4 \cdot 10^{7} 16$-bit samples for each channel. These data are flagged for cosmic-ray events, elevation changes, focal-plane temperature instabilities, and electromagnetic interference events. In general, about $5 \%$ of the data for each channel are flagged and not used in the subsequent analysis. The gaps resulting from this editing are filled with a constrained realization of noise in order to minimize their effect in the subsequent filtering of the data. The data are deconvolved by the bolometer and electronics transfer functions to recover uniform gain at all frequencies.

The noise power spectrum of the data and the maximum-likelihood maps ${ }^{26-28}$ are calculated using an iterative technique ${ }^{29}$ that separates the sky signal from the noise in the time-ordered data. In this process, the statistical weights of frequencies corresponding to angular scales larger than $10^{\circ}$ on the sky are set to zero to filter out the largest-scale modes of the map. The maps are pixelized according to the HEALPix pixelization scheme ${ }^{30}$.

Figure 1 shows the maps obtained in this way at each of the four frequencies. The $400 \mathrm{GHz}$ map is dominated by emission from interstellar dust that is well correlated with that observed by the IRAS and COBE/DIRBE satellites. The 90, 150 and $240 \mathrm{GHz}$ maps are dominated by degree-scale structures that are resolved with high signal-to-noise ratio. A qualitative but powerful test of the hypothesis that these structures are CMB anisotropy is provided by subtracting one 
map from another. The structures evident in all three maps disappear in both the $90-150 \mathrm{GHz}$ difference and in the 240-150 GHz difference, as expected for emission that has the same spectrum as the CMB dipole anisotropy used to calibrate the maps.

To quantify this conclusion, we performed a "colour index" analysis of our data. We selected the $\sim 1800014^{\prime}$ pixel at galactic latitude $b<-15^{\circ}$, and made scatter plots of $90 \mathrm{GHz}$ versus $150 \mathrm{GHz}$ and $240 \mathrm{GHz}$ versus $150 \mathrm{GHz}$. A linear fit to these scatter plots gives slopes of $1.00 \pm 0.15$ and $1.10 \pm 0.16$, respectively (including our present $10 \%$ calibration error), consistent with a CMB spectrum. For comparison, free-free emission with spectral index -2.35 would produce slopes of 2.3 and 0.85 , and is therefore rejected with $>99 \%$ confidence; emission from interstellar dust with temperature $T_{d}=15 \mathrm{~K}$ and spectral index of emissivity $\alpha=1$ would produce slopes of 0.40 and 2.9. For any combination of $T_{d}>7 \mathrm{~K}$ and $1<\alpha<2$, the dust hypothesis is rejected with $>99 \%$ confidence. We conclude that the dominant source of structure that we detect at 90, 150 and $240 \mathrm{GHz}$ is CMB anisotropy.

We further argue that the $150 \mathrm{GHz}$ map at $b<-15^{\circ}$ is free of significant contamination by any known astrophysical foreground. Galactic synchrotron and free-free emission is negligible at this frequency ${ }^{31}$. Contamination from extra-galactic point sources is also small ${ }^{32}$; extrapolation of fluxes from the PMN survey ${ }^{33}$ limits the contribution by point sources (including the three above-mentioned radio-bright sources) to the angular power spectrum derived below to $<0.7 \%$ at $\ell=200$ and $<20 \%$ at $\ell=600$. The astrophysical foreground that is expected to dominate at $150 \mathrm{GHz}$ is thermal emission from interstellar dust. We placed a quantitative limit on this source of contamination as follows. We assumed that dust properties are similar at high $\left(b<-20^{\circ}\right)$ and moderate $\left(-20^{\circ}<b<-5^{\circ}\right)$ Galactic latitudes. We selected the pixels at moderate Galactic latitudes and correlated the structure observed in each of our four bands with the IRAS/DIRBE map, which is dominated by dust in cirrus clouds. The best-fit slope of each of the scatter plots measures the ratios of the dust signal in the BOOMERanG channels to the dust signal in the IRAS/DIRBE map. We found that the $400 \mathrm{GHz}$ map is very well correlated to the IRAS/DIRBE map, and that dust at $b<-20^{\circ}$ ) can account for at most $10 \%$ of the signal variance at $240 \mathrm{GHz}$, 
$3 \%$ at $150 \mathrm{GHz}$ and $0.5 \%$ at $90 \mathrm{GHz}$.

\section{Angular Power Spectra}

We compared the angular power spectrum of structures evident in Fig.1 with theoretical predictions. In doing so, we separated and removed the power due to statistical noise and systematic artifacts from the power due to CMB anisotropies in the maps. The maximumlikelihood angular power spectrum of the maps was computed using the MADCAP ${ }^{34}$ software package, whose algorithms fully take into account receiver noise and filtering.

Full analysis of our entire data set is under way. Because of the computational intensity of this process, we report here the results of a complete analysis of a limited portion of the data chosen as follows. We analysed the most sensitive of the $150 \mathrm{GHz}$ detectors. We restricted the sky coverage to an area with $R A>70^{\circ}, b<-20^{\circ}$ and $-55^{\circ}<D e c<-35^{\circ}$, and we used only

the $\sim 50 \%$ of the data from this detector that was obtained at a scan speed of $1^{\circ} \cdot \mathrm{s}^{-1}$. We used a relatively coarse pixelization of 8,000 14-arcmin pixels as a compromise between computation speed and coverage of high multipoles. Finally, we limited our analysis to $\ell \leq 600$ for which the effects of pixel shape and size and our present uncertainty in the beam size $\left(1^{\prime}\right)$ are small and can be accurately modelled.

The angular power spectrum determined in this way is shown in Fig. 2 and reported in Table 1 . The power spectrum is dominated by a peak at $\ell_{\text {peak }} \approx 200$, as predicted by inflationary cold dark matter models. These models additionally predict the presence of secondary peaks. The data at high $\ell$ limit the amplitude, but do not exclude the presence, of a secondary peak. The errors in the angular power spectrum are dominated at low multipoles $(\ell \leq 350)$ by the cosmic/sampling variance, and at higher multipoles by detector noise.

The CMB angular power spectrum shown in Fig.2 was derived from 4.1 days of observation. As a test of the stability of the result, we made independent maps from the first and second halves of these data. The payload travels several hundred kilometers, and the Sun moves $2^{\circ}$ on the sky, 
between these maps. Comparing them provides a stringent test for contamination from sidelobe pickup and thermal effects. The angular power spectrum calculated for the difference map is shown in Fig.2. The reduced $\chi^{2}$ of this power spectrum with respect to zero signal is 1.11 (12 degrees of freedom), indicating that the difference map is consistent with zero contamination.

\section{A peak at $\ell \approx 200$ implies a flat Universe}

The location of the first peak in the angular power spectrum of the CMB is well measured by by this data setthe BOOMERanG data set. From a parabolic fit to the data at $\ell=50$ to 300 in the angular power spectrum, we find $\ell_{\text {peak }}=(197 \pm 6)(1 \sigma$ error $)$. The parabolic fit does not bias the determination of the peak molutpolemultipole: applying this method to Monte Carlo realizations of theoretical power spectra we recover the correct peak location for a variety of cosmological models. Finally, Notice the peak location is independent of the details of the data calibration, which obviously affect only the height of the peak and not its location. The height of the peak is $\Delta T_{200}=(69 \pm 4) \pm 7 \mathrm{mK}(1-\sigma$ statistical and calibration errors, respectively $)$.

The data are inconsistent with current models based on topological defects (see, for example, ref. 35) but are consistent with a subset of cold dark matter models. We generated a database of cold dark matter models ${ }^{36,37}$, varying six cosmological parameters (the range of variation is given in parentheses): the non relativistic matter density, $\Omega_{m}(0.05-2)$; the cosmological constant, $\Omega_{\Lambda}(0-1)$; the Hubble constant, $\mathrm{h}$ (0.5-0.8); the baryon density, $\mathrm{h}^{2} \Omega_{b}(0.013-0.025)$, the primordial scalar spectral index, ns (0.8-1.3); and the overall normalization A (freeparameter) of the primordial density fluctuation power spectrum. We compared these models with the power spectrum we report here to place constraints on allowed regions in this 6-parameter space. In Figure 3 we mark with black dots the region of the $\Omega_{m}-\Omega_{\Lambda}$ plane where some combination of the remaining four parameters within the ranges defined by our model space gives a power spectrum consistent with our $95 \%$ confidence interval for $\ell_{\text {peak }}$. This region is quite narrow and elongated along the "flat Universe" line $\Omega_{m}+\Omega_{\Lambda}=1$. The width of this region is determined by degeneracy

in the models, which produce closely similar spectra for different values of the parameters ${ }^{38}$. We 
further evaluated the likelihood of the models given the BOOMERanG measurement and the same priors (constraints on the values of the cosmological parameters) as in ref. 16. Marginalizing over all the other parameters, we found the following $95 \%$ confidence interval for $\Omega_{0}=\Omega_{m}+\Omega_{\Lambda}$ : $0.88<\Omega_{0}<1.12$. This provides evidence for a euclidean geometry of the Universe. Our data clearly show the presence of power beyond the peak at $\ell=197$, corresponding to smaller-scale structures. The consequences of this fact will be fully analyzed elsewhere.

\section{Acknowledgments}

The BOOMERanG experiment has been supported by Programma Nazionale di Ricerche in Antartide, Universitá di Roma "La Sapienza", and Agenzia Spaziale Italiana in Italy, by NSF and NASA in the USA, and by PPARC in the UK. We would like to thank the entire staff of

the National Scientific Ballooning Facility, and the United States Antarctic Program personnel in McMurdo for their excellent preflight support and a marvelous LDB flight. Doe/NERSC provided the supercomputing facilities.

Correspondence and request for materials should be addressed to P.d.B. (e-mail: debernardis@roma1.infn.it). Details on the experiment and numerical data sets are available at the web sites (http://oberon.roma1.infn.it/boomerang) and (http://www.physics.ucsb.edu/ boomerang). 
TABLE 1: Angular power specrtum of CMB anisotropy

\begin{tabular}{|c|c|c|}
\hline$\ell$-range & $\begin{array}{c}150 \mathrm{GHz} \\
\left(\left[1^{\text {st }} \text { half }\right]+\left[2^{\text {nd }} \text { half }\right]\right) / 2\end{array}$ & $\begin{array}{c}150 \mathrm{GHz} \\
\left(\left[1^{\text {st }} \text { half }\right]-\left[2^{\text {nd }} \text { half }\right]\right) / 2\end{array}$ \\
\hline$[26-75]$ & $1140 \pm 280$ & $63 \pm 32$ \\
\hline$[76-125]$ & $3110 \pm 490$ & $16 \pm 20$ \\
\hline$[126-175]$ & $4160 \pm 540$ & $17 \pm 28$ \\
\hline$[176-225]$ & $4700 \pm 540$ & $59 \pm 44$ \\
\hline$[226-275]$ & $4300 \pm 460$ & $68 \pm 59$ \\
\hline$[276-325]$ & $2640 \pm 310$ & $130 \pm 82$ \\
\hline$[326-375]$ & $1550 \pm 220$ & $-7 \pm 92$ \\
\hline$[376-425]$ & $1310 \pm 220$ & $-60 \pm 120$ \\
\hline$[426-475]$ & $1360 \pm 250$ & $0 \pm 160$ \\
\hline$[476-525]$ & $1440 \pm 290$ & $220 \pm 230$ \\
\hline$[526-575]$ & $1750 \pm 370$ & $130 \pm 300$ \\
\hline$[576-625]$ & $1540 \pm 430$ & $-430 \pm 360$ \\
\hline
\end{tabular}

Shown are measurements of the angular power spectrum of the cosmic microwave background at $150 \mathrm{GHz}$, and test for systematic effects. The values listed are for $\Delta T_{\ell}^{2}=\ell(\ell+1) c_{\ell} / 2 \pi$, in $\mathrm{mK}^{2}$. Here $c_{\ell}=<a_{\ell m}^{2}>$, and $\left\langle a_{\ell m}^{2}>\right.$ are the coefficients of the spherical harmonic decomposition of the CMB temperature field: $\Delta T(\theta, \phi)=\sum a_{\ell m} Y_{\ell m}(\theta, \phi)$. The stated $1 \sigma$ errors include statistical and cosmic/sample variance, and do not include a $10 \%$ calibration uncertainty. 
Figure 1: BOOMERanG sky maps (equatorial coordinates). The sky maps at 90, 150, and $240 \mathrm{GHz}$ (left panels) are shown with a common color scale, using a thermodynamic temperature scale chosen such that CMB anisotropies will have the same amplitude in the three maps. Only the colour scale of the $400 \mathrm{GHz}$ map (bottom right) is 14 times larger than the others: this has been done to facilitate comparison of emission from interstellar dust (ISD), which dominates this map, with ISD emission present in the lower-frequency maps. The maps at 90 and $400 \mathrm{GHz}$ are each from a single detector, while maps at 150 and $240 \mathrm{GHz}$ have each been obtained by co-adding data from three detectors. For purposes of presentation, the maps have been smoothed with gaussian filters to obtain FWHM effective resolution of $22.5^{\prime}$ (small circle in the bottom right side of each panel). Structures along the scan direction larger than $10^{\circ}$ are not present in the maps. Several features are immediately evident. Most strikingly, the maps at 90, 150, and $240 \mathrm{GHz}$ are dominated by degreescale structures that fill the map, have well-correlated morphology and are identical in amplitude in all three maps. These structures are not visible at $400 \mathrm{GHz}$. The $400 \mathrm{GHz}$ map is dominated by diffuse emission which is correlated with the ISD emission mapped by IRAS/DIRBE ${ }^{22}$. This emission is strongly concentrated towards the right-hand edge of the maps, near the plane of the Galaxy. The same structures are evident in the 90,150 and $240 \mathrm{GHz}$ maps at galactic latitude $b>-15^{\circ}$, albeit with an amplitude that decreases steeply with decreasing frequency.

The large-scale gradient evident especially near the right edge of the $240 \mathrm{GHz}$ map is a result of high-pass filtering the very large signals near the Galactic plane (not shown). This effect is negligible in the rest of the map. The two top right panels show maps constructed by differencing the 150 and $90 \mathrm{GHz}$ maps and the 240 and $150 \mathrm{GHz}$ maps. The difference maps contain none of the structures that dominate the maps at 90, 150 and $240 \mathrm{GHz}$, indicating that these structures do indeed have the ratios of brightness that are unique to the CMB. The morphology of the residual structures in the $240-150 \mathrm{GHz}$ map is well-correlated with the $400 \mathrm{GHz}$ map, as is expected if the residuals are due to the ISD emission. Three compact sources of emission are visible in the lower-frequency maps, as indicated by the circles. These are known radio-bright quasars from the SEST pointing catalogue at $230 \mathrm{GHz}$. The boxed area has been used for computing the angular power spectrum shown in Fig.2. 
Figure 2: Angular power spectrum measured by BOOMERanG at $150 \mathrm{GHz}$. Each point is the power averaged over $\Delta \ell=50$ and has negligible correlations with the adjacent points. The error bars indicate the uncertainty due to noise and cosmic/sampling variance. The errors are dominated by cosmic/sampling variance at $\ell<350$; they grow at large $\ell$ due to the signal attenuation caused by the combined effects ${ }^{39}$ of the $10^{\prime}$ beam and the $14^{\prime}$ pixelization $(0.87$ at $\ell=200$ and 0.33 at $\ell=600)$. The current $\pm 10 \%$ uncertainty in the calibration corresponds to an overall re-scaling of the $\mathrm{y}$-axis by $\pm+20 \%$, and is not shown. The current $1^{\prime}$ uncertainty in the angular resolution of the measurement creates an additional uncertainty, indicated by the distance between the ends of the red error bars and the blue horizontal lines, that is completely correlated and is largest (11\%) at $\ell=600$. The green points show the power spectrum of a difference map obtained dividing the data in two parts corresponding to the first and second halves of the timestream. We make two maps (A and B) from these halves, and the green points show the power spectrum computed from the difference map, (A-B)/2. Signals originating from the sky should disappear in this map, so this is a test for contamination in the data (see text). The solid curve has parameters $\left(\Omega_{b}, \Omega_{m}, \Omega_{\Lambda}\right.$, $\mathrm{ns}, \mathrm{h})=(0.05,0.31,0.75,0.95,0.70)$. It is the best fit model for the BOOMERanG test flight data $^{15,16}$, and is shown for comparison only. The model that best fits the new data reported here will be presented elsewhere.

Figure 3: Observational constraints on $\Omega_{m}$ and $\Omega_{\Lambda}$. All the cosmological models (from our data base) consistent with the position of the peak in the angular power spectrum measured by BOOMERanG (95\% confidence intervals) define an "allowed" region in the $\Omega_{m}-\Omega_{\Lambda}$ plane (dotted region). Such a region is elongated around the $\Omega_{0}=1$ line identifying a flat geometry, Euclidean Universe. The blue lines define the age of the Universe for the considered models. The green shaded region is consistent (95\% confidence contour) with the recent results of the high-redshift supernovae surveys ${ }^{40,41}$. 


\section{References}

1. Sachs R.K., Wolfe, A.M., Perturbations of a Cosmological model and angular variations of the microwave background, Astrophys.J, 147, 73-90 (1967)

2. Weinberg, Gravitation and Cosmology, John Wiley \& Sons, New York (1972)

3. Hu W., Sugiyama N., Silk J., The physics of cosmic microwave background anisotropies, Nature, 386, 37-43 (1997)

4. Bond J.R., Efstathiou G., Tegmark M., Forecasting cosmic parameter errors from microwave background anisotropy experiments, MNRAS 291, L33-L41, (1997)

5. Hinshaw G., Banday A.J., Bennett C.L., Gorski K.M., Kogut A., Smoot G.F., and Wright E.L., Band power spectra in the COBE-DMR four-year anisotropy map, Astrophys. J. 464, L17-L20, (1996)

6. Scott P.F. et al., Measurement of structure in the Cosmic Background Radiation with the Cambridge Cosmic Anisotropy Telescope Astrophys.J.Letters, 461, L1-L4 (1996)

7. Netterfield C.B., et al., A Measurement of the Angular Power Spectrum of the Anisotropy in the Cosmic Microwave Background, Astrophys.J., 474, 47-66 (1997)

8. Leitch E.M., et al., A Measurement of Anisotropy in the Cosmic Microwave Background on 7-22 Arcminute Scales, Astrophys.J., submitted, astro-ph/ 9807312 (1998)

9. Wilson G.W., et al., New CMB Power Spectrum Constraints from MSAMI, Astrophys.J., submitted, astro-ph/9902047, (1999)

10. Baker J.C., et al., Detection of Cosmic Microwave Background structure in a second field with the Cosmic Anisotropy Telescope, MNRAS submitted, astro-ph/9904415, (1999)

11. Peterson J.B., et al., First Results from Viper: Detection of Small-Scale Anisotropy at 40 GHZ, astro-ph/9910503 (1999)

12. Coble, K., et al., Anisotropy in the Cosmic Microwave Background at Degree Angular Scales: Python V Results, Astrophys.J.Letters 519, L5, (1999) 
13. Torbet, E., et al., A Measurement of the Angular Power Spectrum of the Microwave Background Made from the High Chilean Andes, Astrophys.J.Letters, 521, 79-82 (1999)

14. Miller A.D., et al., A Measurement of the Angular Power Spectrum of the CMB from $=100$ to 400, Astrophys.J., submitted, astro-ph / 9906421 (1999)

15. Mauskopf P., et al., Measurement of a peak in the CMB power spectrum from the test flight of BOOMERanG, Astrophys.J., submitted, astro-ph/9911444 (1999)

16. Melchiorri A. et al, A measurement of $\Omega$ from the North American test flight of BOOMERanG, Astrophys.J., submitted, astro-ph/9911445 (1999)

17. de Bernardis P. et al., Mapping the CMB sky : the BOOMERanG experiment, New Astronomy Reviews, 43, 289-296, (1999)

18. Mauskopf P. et al., Composite Infrared Bolometers with Si3N4 Micromesh Absorbers, Applied Optics 36, 765-771, (1997)

19. Bock. J., et al., Silicon nitride micromesh bolometer arrays for SPIRE, in Proc. SPIE 3357, 297-304, (1998) Advanced Technology MMW, Radio, and Terahertz Telescopes, Thomas G. Phillips; Ed.

20. Masi S., et al., A self contained ${ }^{3}$ He refrigerator suitable for long duration balloon experiments, Cryogenics, 38, 319-324 (1998).

21. Masi S., et al., A long duration cryostat suitable for balloon borne photometry, Cryogenics, 39, 217-224 (1999).

22. Schlegel D.J., Finkbeiner D.P., \& Davis M., Maps of Dust IR Emission for Use in Estimation of Reddening and CMBR Foregrounds, Astrophys.J. 500, 525-553 (1998)

23. Delabrouille J., Gorski, K.M., Hivon E., Circular scans for CMB anisotropy observation and analysis, MNRAS, 298, 445-450 (1998)

24. Cheung L.H., et al, 1.0 millimeter maps and radial density distributions of southern HII/Molecular Cloud Complexes, Astrophys.J., 240, 74-83 (1980). 
25. A. Kogut, et al., Dipole anisotropy in the COBE DMR first-year sky maps, Astrophys.J., 419, 1-6 (1993)

26. Tegmark M., CMB mapping experiments: a designer's guide, Phys.Rev. D56, 4514-4529 (1997)

27. J.R. Bond, R.Crittenden, A.H. Jaffe \& L.E.Knox, Computing challenges of the Cosmic Microwave Background, Computing in Science and Engineering, 21, 1, 1999. (astro$\mathrm{ph} / 9903166)$

28. Borrill J., The challenge of data analysis for future CMB observations, Proc. of the 3K Cosmology EC-TMR conference, eds. L. Maiani, F. Melchiorri, N. Vittorio, AIP CP 476, $277,(1999)$.

29. Prunet S., et al., Iterative Map-Making for scanning experiments, Proc. of the conference "Energy density in the Universe" D. Langlois, R. Ansari, J. Bartlett, editors, editiones Frontieres, (2000)

30. Gorski K.M., Hivon E. \& Wandelt B.D., Analysis Issues for Large CMB Data Sets, 1998, Proceedings of the MPA/ESO Conference, Garching 2-7 August 1998, eds. A.J. Banday, R.K. Sheth and L. Da Costa, (astro-ph / 9812350); see also http://www.tac.dk healpix/

31. Kogut A., Anomalous Microwave Emission in Microwave Foregrounds, in Microwave Foregrounds, Eds. A. de Oliveira Costa and M. Tegmark, Astron. Soc. Pacific conf. Series 181, 91-99, (1999)

32. Toffolatti L., et al., Extragalactic source counts and contributions to the anisotropies of the CMB, MNRAS, 297, 117-127 (1998)

33. http://astron.berkeley.edu/wombat/foregrounds/radio.html

34. MADCAP: The Microwave Anisotropy Dataset Computational Analysis Package' Julian Borrill in "Proceeedings of the 5th European SGI/Cray MPP Workshop" (1999). astro-ph/9911389; see also http://cfpa.berkeley.edu borrill/cmb/madcap.html 
35. Durrer R., Kunz M., Melchiorri A., Phys.Rev. D59 (1999) 123005-(1-26) and references therein.

36. Seljak U., Zaldarriaga M., A Line of Sight Approach to Cosmic Microwave Background Anisotropies, Astrophys.J. , 469, 437, (1996)

37. Lewis A., Challinor A., Lasenby A., Efficient Computation of CMB anisotropies in closed FRW models, astro-ph/9911177 (1999)

38. Efstathiou G., and Bond R., Cosmic confusion: degeneracies among cosmological parameters derived from measurements of microwave background anisotropies, MNRAS, 304, 75-97, (1998)

39. Wright E., et al, Comments on the statistical analysis of excess variance in the COBE-DMR maps, Astrophys.J., 420, 1-8 (1994)

40. Perlmutter S., et al., Measurements of $\Omega$ and $\Lambda$ from 42 high-redshift supernovae, Astrophys.J., 517, 565-586, 1999.

41. Schmidt B.P., et al., The High-Z supernova search: measuring cosmic deceleration and global curvature of the Universe using type Ia supernovae, Astrophys.J., 507, 46-63, 1998. 
This figure "fig1.jpg" is available in "jpg" format from: http://arXiv.org/ps/astro-ph/0004404 


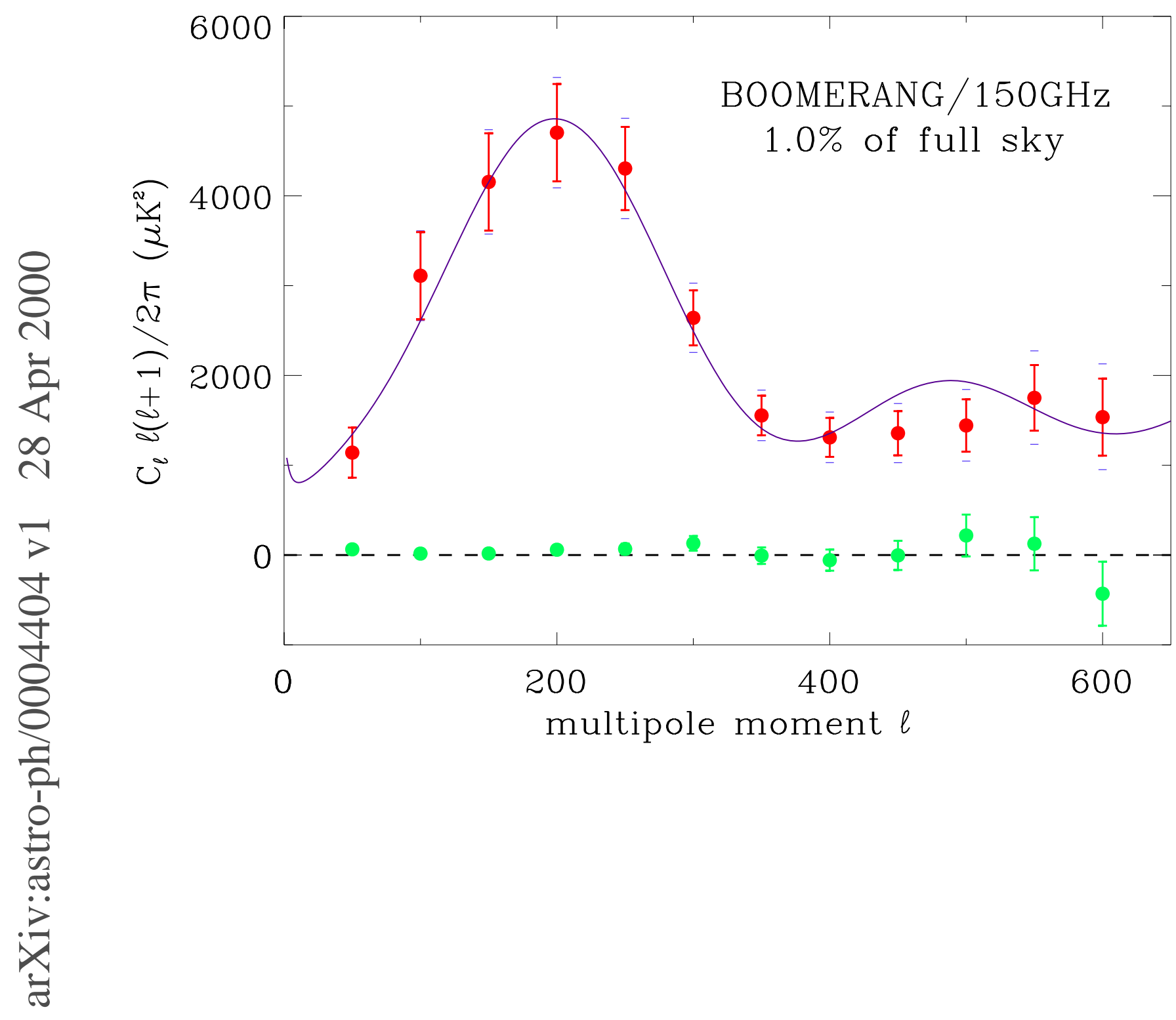



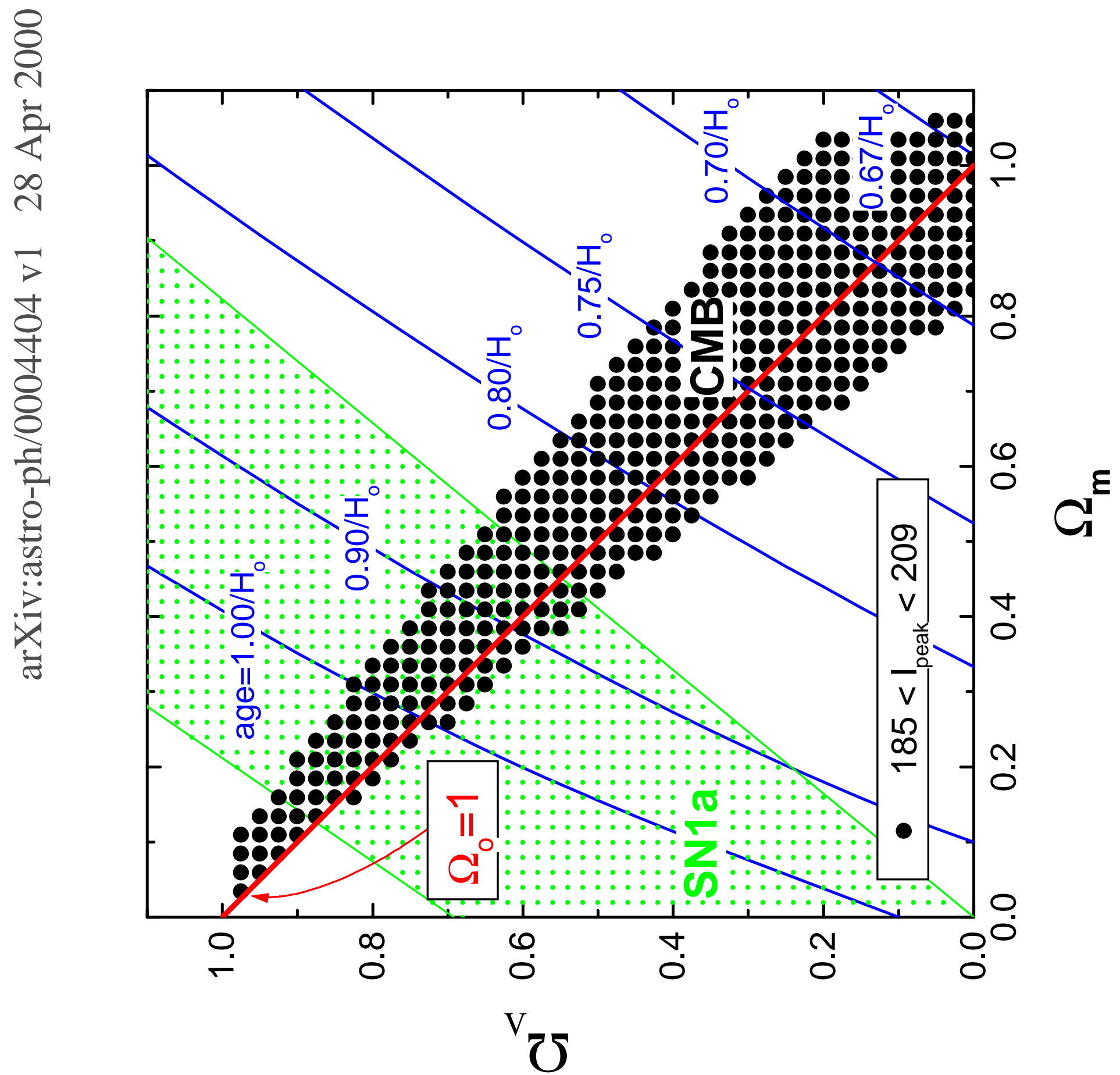